\title{
High levels of mannose-binding lectin are associated with lower pulse wave velocity in uraemic patients
}

\author{
Mads Hornum ${ }^{1 *}$, Jakob T Bay², Peter Clausen ${ }^{1}$, Jesper Melchior Hansen ${ }^{3}$, Elisabeth R Mathiesen ${ }^{4}$,
} Bo Feldt-Rasmussen ${ }^{1}$ and Peter Garred ${ }^{2}$

\begin{abstract}
Background: Uraemia is associated with a highly increased risk of cardiovascular disease. Mannose-binding lectin (MBL) has been shown to be involved in cardiovascular pathophysiology and a protective effect of MBL is suggested. The purpose of the present study was to evaluate a potential impact of MBL on vascular parameters in uraemic patients.

Methods: A cohort of 98 patients with end stage renal disease (ESRD) awaiting kidney transplantation had pulse wave velocity (PWV) and augmentation index (AIX) examined by tonometry and endothelial dependent flow-mediated (FMD) and endothelial independent nitroglycerin-induced (NID) dilatory capacities of the brachial artery measured by ultrasound. An oral glucose tolerance test (OGTT) was performed and serum levels of MBL were measured using Luminex xMAP bead array technology.

Results: The cohort was divided in two groups according to MBL-concentration below or above the median concentration. These groups were comparable regarding age, BMI, and duration of ESRD. PWV was significantly lower in the group with high MBL levels compared to the group with low MBL levels and trends toward better AIX and higher insulin sensitivity (ISI) was also seen in the group with high MBL levels. No difference was seen in FMD and NID.

Conclusions: High levels of MBL are associated with lower PWV and the use of antihypertensive drugs in a cohort of patients with ESRD awaiting kidney transplantation suggesting a beneficial role of high levels of MBL on arterial stiffness in uraemia.
\end{abstract}

Keywords: Arterial stiffness, Augmentation index, Endothelial function, Glucose intolerance, Mannose-binding lectin

\section{Background}

Uraemia is associated with a highly increased risk of cardiovascular disease. Vascular function in uraemic patients is severely damaged by a number of factors including increased phosphate, hypertension and increased calcification of large arteries. Stiffness of elastic large arteries as measured by pulse wave velocity are associated with increased mortality $[1,2]$. Hypertension and age seems to be especially important for the development of arterial stiffness in non-

\footnotetext{
* Correspondence: mads.hornum@regionh.dk

'Department of Nephrology, Copenhagen University Hospital, Rigshospitalet, Copenhagen, Denmark

Full list of author information is available at the end of the article
}

diabetic uraemic patients [3]. Other factors like inflammation, infections and hyperlipidaemia damage the endothelial function as measured by flow mediated vasodilatation in the brachial artery $[4,5]$. Genetically determined variation in MBL- serum concentration has been shown to affect the clinical course of cardiovascular disease, and an impairment of endothelial function caused by low MBL concentrations has been described in childhood infection [6]. Potential effects of MBL concentrations on arterial stiffness are not well described and especially the potential influence of MBL in the deterioration of arterial and endothelial function in uraemia needs further investigation. 
The aim of the present study was to evaluate a potential impact of MBL on vascular parameters in uraemic patients.

\section{Methods}

This is a prospective, observational cohort study including 98 patients with a scheduled living donor kidney transplantation $(n=48)$ or deceased donor transplantation on the waiting list for transplantation $(n=50)$ in the period between January 2006 and March 2008 at the two transplantation centres in Copenhagen (Copenhagen University Hospital, Rigshospitalet and Herlev Hospital (Tx-group)). To be included the patients had to be between 18 to 65 years of age with no more than one previous kidney transplantation.

The Scientific-Ethical Committee of the Capital Region of Denmark (\# KF 01279825) and The Data Protection Agency (\#2006-41-5640) approved the study. All participants gave their informed written consent.

\section{Study procedure}

All participants were examined before kidney transplantation. The majority of the patients were anuric and the few with remaining diuresis all had an estimated baseline creatinine clearance below $15 \mathrm{ml} / \mathrm{min}$. The examinations were performed after an overnight fast including coffee, tobacco, and exercise abstinence for 10 hours. Antihypertensive medication was allowed in the morning. The examiners were blinded regarding the actual clinical and metabolic status of the patient at the time of examination and all clinical information was analyzed and described after data collection was completed. Data regarding duration of smoking history, ESRD, antihypertensive treatment were obtained by interview and from the medical record. Weight and height were measured.

Hemodialysis patients were examined between days of hemodialysis. Peritoneal dialysis patients had the peritoneal fluid (PF) drained in the morning at 6 a.m. The OGTT was performed more than 12 hours after last instillation of PF and more than 4 hours after draining the PF. After 10 minutes in the supine resting position blood pressure was measured in the arm opposite to a fistula or dialysis catheter. Mean arterial blood pressure (MAP) was given as mean of three measurements. Fasting blood samples were drawn in an antecubital vein from the same arm for the determination of plasma (p)-glucose (PG), p-CRP and p-homocystein. PG concentrations were analysed by the glucose-hexokinase method (Gluco-quant ${ }^{\oplus}$, Roche Diagnostics GmbH, D-68298 Mannheim, Germany) and p-insulin were measured using enzyme-linked immunosorbent assay kits (Elecsys, Roche Diagnostics GmbH, D68298 Mannheim, Germany). All assays were automated and performed on a Cobas Fara robot (Roche Diagnostics GmbH, Mannheim, Germany).

\section{Measurement of serum MBL}

Serum MBL concentrations were measured using an antiMBL monoclonal antibody double sandwich technique based on the Luminex xMAP bead array technology as previously described [7]. In healthy subjects the median dayto-day variability in $\mathrm{MBL}$ concentrations expressed as $\mathrm{CV}$ was $6 \%$. The lower detection level was $5 \mu \mathrm{g} / \mathrm{L}$ and intraand interassay CVs were below 5 and $10 \%$, respectively.

\section{MBL2 genotypes}

Blood collected in $10 \mathrm{ml}$ EDTA containing vacutainers was used for DNA extraction. Anticoagulated blood was frozen at $-80^{\circ} \mathrm{C}$ before DNA processing. MBL2 single nucleotide polymorphisms (SNPs) in form of the structural variants named B (codon 54, rs1800450, C (codon 57, rs1800451, and D (codon 52, rs5030737) were typed by PCR using sequence specific priming (PCR-SSP) as previously described [8]. The normal allele is designated " $A$ ". All three structural variant alleles $(B, C$, and $D)$ have a considerable effect on MBL concentrations and to avoid small groups, the three alleles were grouped in one category called allele " $O$ " for statistical analyses.

Standard laboratory methods were applied for the analysis of the other samples.

\section{Pulse wave analysis and velocity}

PWV and AIX of the carotid-femoral pulse wave was recorded sequentially at the carotid and femoral artery using applanation tonometry (SphygmoCor ${ }^{\oplus}$; AtCor Medical, Sydney, NSW, Australia) performed by the same observer $(\mathrm{MH})$ as previously described in detail [3]. The reproducibility of AIX and PWV for two repeat scans on two different days in ten uraemic patients from the present study was analysed using the Bland Altman method. The mean $\pm \mathrm{SD}$ intra-observer difference was $0.1 \pm 0.7 \mathrm{~m} / \mathrm{s}$ for PWV, $1.7 \pm 5.5 \%$ for AIX, which is in accordance with other groups $[4,9]$.

\section{Flow mediated vasodilatation (FMD) and nitroglycerin-induced dilatation (NID)}

An Acuson $128 \mathrm{XP} / 10^{\mathrm{m}}$ ultrasound system with extended frequency-imaging option and a $7-\mathrm{MHz}$ linear transducer was used to measure the brachial artery dilatory responses to increased flow (FMD) and to nitroglycerin (NID) based on previous published methodology by our group and others $[4,9,10]$.

External ultrasound measurements of FMD and NID have previously been shown to provide accurate and reproducible results. Coefficients of variation of the diameter changes expressed as diameter in percentage of the baseline scan diameter (100\%) in the present study was 4.4 and 4.1\% for FMD and NID for eight scans of the same subject from the present study population (within patient variability) performed on separate days within a period of 
2 weeks by the same investigator, similar to previous reports $[9,11]$.

\section{Evaluation of glucose tolerance}

A 75-gram OGTT was performed according to the WHO criteria [12] and an insulin sensitivity index (ISI) was calculated as previously described in details [13].

\section{Antihypertensive treatment}

Antihypertensive treatment mainly included beta-blockade, calcium channel blockade, angiotensin-II-blockade and diuretics.

\section{Statistics}

Data analyses were performed using Statistical Analysis Software $\left(\mathrm{SAS}^{\circ}\right)$ version 9.1. Unless specified otherwise, continuous data is described as mean \pm standard deviation (SD) or median and range for normal and skewed distributions, respectively. The Shapiro-Wilk W test and a normal plot were used to judge normality. Group comparisons of continuous data were performed using unpaired t-test test for normally distributed data or non-parametric Wilcoxon Rank Sum Test or Kruskal-Wallis non-parametric one way analysis of variance test. Chi square or Fisher's exact tests were used for group comparisons between categorical data where appropriate. A p value of $<0.05$ was used to determine significance.

\section{Results}

The cohort was divided in two groups according to MBL concentration below or above the median concentration. These groups were comparable regarding age, hypertension, glycemic status, BMI, and duration of ESRD.

Univariate analysis showed no association between the MBL level and PWV, ISI, FMD, NID or augmentation index in the whole group but the variance analysis between the two groups demonstrated that PWV was significantly lower in the group with high MBL levels compared to the group with low MBL levels (Table 1, $\mathrm{p}=0.03$ ) and trends toward better AIX and higher ISI was also seen in the group with high MBL levels. No difference was seen in FMD or NID. Systolic and diastolic blood pressure was similar between groups, however the use of antihypertensive drugs were significantly higher in the group with the lowest PWV and highest MBL (Table 1).

Data on myocardial infarction, stroke and smoking were collected and there was no difference between the groups (Table 1).

The variance analysis was also performed for the whole group with factors known to influence PWV, describing $65 \%$ of the variance and as expected age, hypertension, glucose intolerance, insulin resistance and homocystein was significantly associated with PWV, but MBL levels just did not reach statistical significance in this analysis (Table 2).

Distribution of the genetic MBL2-variants differed as result of the stratification according to MBL below or above the median. The normal $A / A$ genotype dominated in the group above the median (44 vs. 5), whereas patients heterozygous for MBL2 variants $(A / O)$ haplotype were predominant in the group below the median (41 vs. 5). Those heterozygous for the structural variants, the $\mathrm{O} / \mathrm{O}$ genotype, were found only in the group below the median (6 vs. 0), (Table 3). The difference in MBL concentrations between the MBL2 variants is shown in Figure 1.

The distribution of haemodialysis or peritoneal dialysis, and the use of an arterio-venous fistulae or a permanently haemodialysis catheter, was determined in the cohort, and there were no statistical difference in PWV, ISI, FMD, NID or augmentation index between the groups divided by MBL levels.

\section{Discussion}

In a cohort of patients with ESRD awaiting kidney transplantation we found that high levels of MBL and increased use of antihypertensive drugs were associated with lower PWV. There may be a beneficial role of high levels of MBL on arterial stiffness in uraemia which was independent of BMI, length of uraemia and age. Previously Cheung et al. [14] demonstrated that the MBL genotype influenced arterial stiffness in Chinese children with Kawasaki Disease. The children with low level MBL expression IL/LLgenotypes without coronary complications had faster PWV/stiffer peripheral conduit arteries than children with high level MBL expression HL- genotypes indicating that a high level of MBL had a protective effect on arterial function. In a study of healthy Danish blood donors aged 18-65 years, the median value of MBL was $967.5 \mathrm{ug} / \mathrm{L}$ (8$4693 \mathrm{ug} / \mathrm{L})$ [7]. Arterial stiffness estimated by PWV [15] reflects damage of the elastic tissue of aorta $[15,16]$ and is associated with increased mortality in ESRD-patients $[17,18]$. In a cohort of 66 uraemic patients we previously reported that age and mean arterial pressure but not prediabetes were independently associated with increased PWV [3] and an age and BMI matched healthy control group previously studied by our group had significant lower blood pressure and a better augmentation index, FMD and NID compared to uraemic patients on the waiting list for Tx [19]. In the present cohort divided by the median level of MBL there was no difference in age, fasting glucose, 2-hour blood glucose, ISI or blood pressure between the groups, however more antihypertensive drugs were used in the group with high MBL levels and the lowest PWV, indicating that the role of MBL in uraemia seems independently of traditional risk factors for an increased PWV. Although not significant, both augmentation index and ISI index was more favourable in the high 
Table 1 Comparison of clinical and demographic data between patients with a MBL level above or below the median

\begin{tabular}{|c|c|c|}
\hline & $\mathrm{MBL}<722 \mathrm{ug} / \mathrm{L}$ & MBL $>722 \mathrm{ug} / \mathrm{L}$ \\
\hline & $N=49$ & $N=49$ \\
\hline Age (years) & $45.3 \pm 13.0$ & $41.6 \pm 12.4$ \\
\hline ESRD (months) & $29(0-168)$ & $33(0-120)$ \\
\hline \multicolumn{3}{|l|}{ Dialysis status: } \\
\hline Haemodialysis (N) & 34 & 32 \\
\hline Peritoneal dialysis (N) & 13 & 13 \\
\hline Pre-dialysis (N) & 2 & 4 \\
\hline Haemodialysis, venous access: Arterio-venous fistulae/permanently haemodialysis catheter ( $N$ ) & $15 / 19$ & $15 / 17$ \\
\hline Tx status (living donor/deceased donor) & $22 / 27$ & $26 / 23$ \\
\hline Myocardial infarction (N) & 2 & 2 \\
\hline Smoking (N) & 15 & 14 \\
\hline Stroke $(\mathrm{N})$ & 3 & 2 \\
\hline Diabetes (N) & 3 & 5 \\
\hline Impaired fasting glucose $(\mathrm{N})$ & 1 & 2 \\
\hline Impaired glucose tolerance $(\mathrm{N})$ & 21 & 13 \\
\hline Normal glucose tolerance $(\mathrm{N})$ & 24 & 29 \\
\hline Fasting plasma glucose (mmol/L) & $5.0 \pm 0.4$ & $5.1 \pm 0.5$ \\
\hline OGTT 120min(mmol/L) & $8.0 \pm 2.1$ & $7.7 \pm 2.1$ \\
\hline ISI (index) & $7.4 \pm 4.1$ & $7.9 \pm 4.9$ \\
\hline BMI $\left(\mathrm{kg} / \mathrm{m}^{2}\right)$ & $24.2 \pm 3.8$ & $24.5 \pm 3.6$ \\
\hline $\mathrm{PWV}(\mathrm{m} / \mathrm{s})$ & $8.2(5.6-15.8)$ & $6.8(5.2-16.4)^{*}$ \\
\hline AIX (\%) & $27(-10 ; 56)$ & $20(-7 ; 50)$ \\
\hline FMD (\%) & $3.8(-8.3 ; 19.2)$ & $3.5(-6.8 ; 19.1)$ \\
\hline NID (\%) & $10.5(2.9 ; 27.1)$ & $11.5(2.0 ; 27.2)$ \\
\hline Systolic blood pressure $(\mathrm{mmHg})$ & $143 \pm 25$ & $140 \pm 19$ \\
\hline Diastolic blood pressure $(\mathrm{mmHg})$ & $85 \pm 12$ & $84 \pm 13$ \\
\hline Number of antihypertensive drugs & $2(0-5)$ & $3(0-6)^{*}$ \\
\hline P-C-reactive protein (mg/L) & $2(1-40)$ & $2(1-21)$ \\
\hline P-Homocystein (umol/L) & $21(11-62)$ & $22(12-150)$ \\
\hline P-PTH (pmol/L) & $21(2-100)$ & $20(4-156)$ \\
\hline P-ionized calcium (mmol/L) & $1.19 \pm 0.09$ & $1.21 \pm 0.09$ \\
\hline P-phosphate (mmol/L) & $1.8 \pm 0.4$ & $2.0 \pm 0.6$ \\
\hline
\end{tabular}

Data are presented as mean (SD) or median and range.

MBL: Mannose Binding Lectin, ESRD: end-stage renal disease,

Tx: transplantation, BMI: body mass index, PTH: parathyroidea hormone,

OGTT: oral glucose tolerance index, ISI: insulin sensitivity index.

PWV: pulse wave velocity,

AIX: Augmentation Index, FMD: flow mediated vasodilatation,

NID: nitroglycerin independent dilatation,

${ }^{*} \mathrm{P}<0.05$.

MBL group, also suggesting a better arterial function in this group, and a more powerful study including more patients might confirm this association.

The duration of ESRD in the groups divided by the median MBL was not significantly different, an important point, since duration of haemodialysis increases MBL levels as demonstrated by Satomura et al. [20].
Apart from influencing arterial stiffness, MBL deficiency is also known to be associated with severe atherosclerosis [21] and doubles the risk for myocardial infarction (MI) in young relatively healthy Caucasians [22], and leads to cardiovascular events in type 2 diabetic South Asians [23]. Furthermore, MBL deficiency has been shown to be unfavourable in type 2 diabetic Caucasians with MI [24]. 
Table 2 Results of an ANOVA of factors obtained at baseline in 98 ESRD patients awaiting kidney transplantation the model describes $65 \%$ of the variance in PWV $(P<0.0001)$

\begin{tabular}{|c|c|c|c|}
\hline Parameter & Effect on $\operatorname{PWV}(\mathrm{m} / \mathrm{s})$ & $95 \%$ confidence intervals & $P$-value \\
\hline Intercept & -0.14 & $-3.66 ; 3.38$ & 0.94 \\
\hline Age (years) & 0.12 & $0.08 ; 0.16$ & $<0.0001$ \\
\hline $\mathrm{BP}>130 / 80 \mathrm{mmHg}$ & 1.76 & $0.81 ; 2.72$ & 0.0005 \\
\hline BP $<130 / 80$ mmHg (reference group) & 0 & & \\
\hline Number of antihypertensives ( $n$ ) & -0.08 & $-0.45 ; 0.29$ & 0.65 \\
\hline ISI (index) & 0.21 & $0.07 ; 0.36$ & 0.0043 \\
\hline OGTT120min & 0.33 & $0.09 ; 0.56$ & 0.0067 \\
\hline Homocystein (umol/L) & 0.03 & $0.01 ; 0.05$ & 0.01 \\
\hline MBL Genotype A/A & 1.23 & $-1.21 ; 3.66$ & 0.32 \\
\hline MBL Genotype A/O & 0.18 & $-1.85 ; 2.21$ & 0.86 \\
\hline MBL Genotype O/O (reference group) & 0 & & \\
\hline LogMBL & -0.88 & $-1.85 ; 0.10$ & 0.0764 \\
\hline Female sex & 0.31 & $-0.81 ; 1.42$ & 0.58 \\
\hline Male sex (reference group) & 0 & & \\
\hline ESRD (months) & -0.01 & $-0.03 ; 0.01$ & 0.22 \\
\hline Augmentation Index & -0.01 & $-0.05 ; 0.03$ & 0.51 \\
\hline CRP (mg/L) & -0.04 & $-0.11 ; 0.04$ & 0.32 \\
\hline
\end{tabular}

Kruskal-Wallis one way of variance analysis of the effect on pulse wave velocity (PWV) using factors obtained at baseline. BP: blood pressure; ISI: insulin sensitivity index; OGTT: oral glucose tolerance test; MBL: mannose-binding lectin; ESRD: end-stage renal disease; CRP: C-reactive protein.

Although these studies all were performed on patients with normal renal function, they demonstrated that especially the combination of genotype $\mathrm{O} / \mathrm{O}$ and impaired glucose metabolism was a hazardous combination with impact on cardiovascular events. In haemodialysis patients low MBL levels has been found to be associated with increased all-cause mortality [25]. Recently it was shown that both low MBL levels and low expressing MBL2 genotypes were associated with graft loss after kidney transplantation in patients without prior detectable HLA immunization [26]. This and other observations support the notion that MBL may be important to maintain cardiovascular homeostasis, particularly during chronic inflammation, including the importance of lectins in the pathogenesis of several inflammatory diseases [27]. Endothelial function has previously been found to be impaired in both uraemia [9] and in diabetes [28] and may reflect presence of vascular disease or early vascular damage, but in the present study we found no difference in FMD or NID between the two groups. Our cohorts had no signs of

Table 3 Genetic MBL2-variants stratified according to MBL levels

\begin{tabular}{lcc}
\hline MBL2-variant & MBL $<\mathbf{7 2 2} \mathbf{u g} / \mathbf{L}$ & MBL $>\mathbf{7 2 2} \mathbf{~ u g} / \mathbf{L}$ \\
\hline A/A & 2 & 44 \\
A/O & 41 & 5 \\
O/O & 6 & 0 \\
\hline
\end{tabular}

infection and only few diabetics in each group, partly explaining the missing difference between groups in FMD and NID. The overall tendency to infection is however increased in uraemic patients, and aggravated by MBL deficiency, with a high burden of dialysis-related infections and risk of bacteraemia during the dialysis sessions potentially leading to damage of the elastic tissue of the arteries and to an increased arterial stiffness thereby increasing the risk of arteriosclerotic vascular disease.

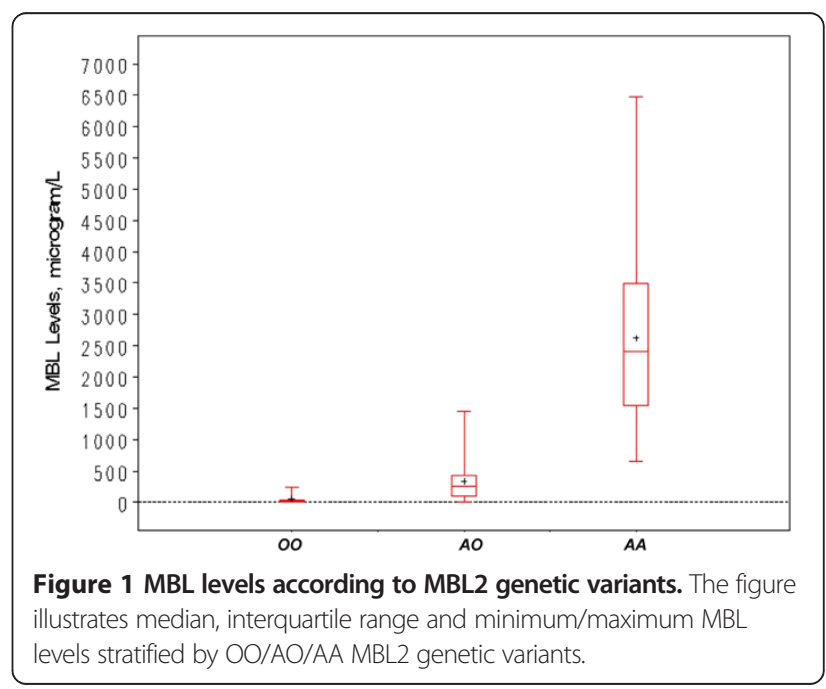


The MBL genotype influences the level of MBL and the AA expression genotype with the highest level of MBL would in theory have the best protection against infections, and thereby the lowest PWV and the highest FMD. This is in accordance with our findings with regard to PWV whereas no effect of MBL on FMD was demonstrated. However, PWV and FMD are not necessarily part in a unified pathophysiological vascular process in uraemia and may be differently influenced by different cardiovascular risk factors such as MBL.

The MBL-genotype A/A clearly was most represented in the high MBL-group in our cohort, potentially indicating a genetic element in the susceptibility to arterial damage in uraemic patients, although the relative few patients with the $\mathrm{OO}$ expression genotype limited the statistical power to detect difference between the groups.

\section{Conclusions}

We demonstrated that high levels of MBL are associated with lower PWV and an increased use of antihypertensive drugs in a cohort of patients with ESRD awaiting kidney transplantation suggesting a beneficial role of high levels of MBL on arterial stiffness in uraemia. We hypothesize that this association will result in a decreased cardiovascular and infection related mortality in patients with high baseline MBL levels and a follow up study is planned to address this hypothesis. The strengths of the study were a carefully examined and characterized cohort, with solid glucose and arterial measurements. Estimation of insulin resistance and validated MBL measurements and determination of genetic MBL2 variants and statistical analysis completed the study. Limitations were the cross sectional design, relatively few patients and lack of follow up data.

\section{Competing interests}

The authors declare that they have no competing interests.

\section{Authors' contributions}

MH: planned, performed and made the statistical analysis and edited and wrote the manuscript. JTB: performed analysis, and wrote the manuscript. PC: planned, performed and wrote the manuscript. JMH: performed the study and wrote the manuscript. ERM: planned the study and wrote the manuscript. BFR: planned, performed and wrote the manuscript. PG: planned, performed analysis and wrote the manuscript. All authors read and approved the final manuscript.

\section{Acknowledgements}

The study was supported by an unrestricted grant from the Danish Kidney Foundation, A. P. Møller Foundation for the Advancement of Medical Science, Eva and Henry Frænkel Foundation, and the Helen Bjørnow Foundation. MH was supported by a fellowship from Rigshospitalet. We thank the laboratory technicians Annette Vinding, Helle Christensen and Andreas Haltorp for their skilful contribution to the data.

\section{Author details}

'Department of Nephrology, Copenhagen University Hospital, Rigshospitalet, Copenhagen, Denmark. 'Laboratory of Molecular Medicine, Department of Clinical Immunology Sect. 7631, Copenhagen University Hospital, Rigshospitalet, Copenhagen, Denmark. ${ }^{3}$ Department of Nephrology, Herlev Hospital, Copenhagen University, Herlev, Denmark. ${ }^{4}$ Department of Endocrinology, Copenhagen University Hospital, Rigshospitalet and University of Copenhagen, Copenhagen, Denmark.
Received: 10 April 2014 Accepted: 25 September 2014

Published: 4 October 2014

\section{Reference}

1. Laurent S, Boutouyrie P, Asmar R, Gautier I, Laloux B, Guize L, Ducimetiere P, Benetos A: Aortic stiffness is an independent predictor of all-cause and cardiovascular mortality in hypertensive patients. Hypertension 2001, 37(5):1236-41.

2. Shoji T, Emoto M, Shinohara K, Kakiya R, Tsujimoto Y, Kishimoto H, Ishimura E, Tabata T, Nishizawa Y: Diabetes mellitus, aortic stiffness, and cardiovascular mortality in end-stage renal disease. J Am Soc Nephrol 2001, 12(10):2117-24.

3. Hornum M, Clausen P, Kjaergaard J, Hansen JM, Mathiesen ER, Feldt-Rasmussen B: Pre-diabetes and arterial stiffness in uraemic patients. Nephrol Dial Transplant 2010, 25(4):1218-25.

4. Celermajer DS, Sorensen KE, Gooch VM, Miller A, Sullivan ID, Lloyd JK, Deanfield JE, Spiegelhalter DJ: Non-invasive detection of endothelial dysfunction in children and adults at risk of atherosclerosis. Lancet 1992, 340(8828):1111-5.

5. Charakida M, Donald AE, Terese M, Leary S, Halcox JP, Ness A, Davey SG Golding J, Friberg P, Klein NJ, Deanfield JE: Endothelial dysfunction in childhood infection. Circulation 2005, 111(13):1660-5.

6. Charakida M, Donald AE, Leary S, Halcox JP, Turner MW, Johnson M, Loukogeorgakis SP, Okorie MI, Davey SG, Deanfield JE, Klein NJ: Endothelial response to childhood infection: the role of mannose-binding lectin (MBL). Atherosclerosis 2010, 208(1):217-21.

7. Bay JT, Garred P: Rapid bead-based immunoassay for measurement of mannose-binding lectin. Scand J Immunol 2009, 69(6):570-5.

8. Garred P, Strom J, Quist L, Taaning E, Madsen HO: Association of mannosebinding lectin polymorphisms with sepsis and fatal outcome, in patients with systemic inflammatory response syndrome. J Infect Dis 2003, 188(9):1394-403.

9. Clausen P, Feldt-Rasmussen B, Iversen J, Lange M, Eidemak I, Strandgaard S: Flow-associated dilatory capacity of the brachial artery is intact in early autosomal dominant polycystic kidney disease. Am J Nephrol 2006, 26(4):335-9

10. Sidhu JS, Newey VR, Nassiri DK, Kaski JC: A rapid and reproducible on line automated technique to determine endothelial function. Heart 2002, 88(3):289-92.

11. Sorensen KE, Celermajer DS, Spiegelhalter DJ, Georgakopoulos D, Robinson J, Thomas O, Deanfield JE: Non-invasive measurement of human endothelium dependent arterial responses: accuracy and reproducibility. Br Heart J 1995, 74(3):247-53.

12. Alberti KG, Zimmet PZ: Definition, diagnosis and classification of diabetes mellitus and its complications. Part 1: diagnosis and classification of diabetes mellitus provisional report of a WHO consultation. Diabet Med 1998, 15(7):539-553.

13. Matsuda M, DeFronzo RA: Insulin sensitivity indices obtained from oral glucose tolerance testing: comparison with the euglycemic insulin clamp. Diabetes Care 1999, 22(9):1462-70.

14. Cheung YF, Ho MH, Ip WK, Fok SF, Yung TC, Lau YL: Modulating effects of mannose binding lectin genotype on arterial stiffness in children after Kawasaki disease. Pediatr Res 2004, 56(4):591-6.

15. O'Rourke MF, Gallagher DE: Pulse wave analysis. J Hypertens Suppl 1996, 14(5):S147-S157.

16. Taniwaki H, Kawagishi T, Emoto M, Shoji T, Kanda H, Maekawa K, Nishizawa $Y$, Morii H: Correlation between the intima-media thickness of the carotid artery and aortic pulse-wave velocity in patients with type 2 diabetes. Vessel wall properties in type 2 diabetes. Diabetes Care 1999, 22(11):1851-7.

17. Blacher J, Guerin AP, Pannier B, Marchais SJ, Safar ME, London GM: Impact of aortic stiffness on survival in end-stage renal disease. Circulation 1999, 99(18):2434-9.

18. London GM, Blacher J, Pannier B, Guerin AP, Marchais SJ, Safar ME: Arterial wave reflections and survival in end-stage renal failure. Hypertension 2001, 38(3):434-8.

19. Hornum M, Clausen P, Idorn T, Hansen JM, Mathiesen ER, Feldt-Rasmussen B: Kidney transplantation improves arterial function measured by pulse wave analysis and endothelium-independent dilatation in uraemic patients despite deterioration of glucose metabolism. Nephrol Dial Transplant 2011, 26(7):2370-7. 
20. Satomura A, Fujita T, Yanai M, Kumasaka K, Uehara Y, Okada K, Fuke $Y$, Nakayama T: Functional mannose-binding lectin levels in patients with end-stage renal disease on maintenance hemodialysis. J Innate Immun 2012, 4(3):293-300.

21. Madsen HO, Videm V, Svejgaard A, Svennevig JL, Garred P: Association of mannose-binding-lectin deficiency with severe atherosclerosis. Lancet 1998, 352(9132):959-60.

22. Vengen IT, Madsen HO, Garred P, Platou C, Vatten L, Videm V: Mannosebinding lectin deficiency is associated with myocardial infarction: the HUNT2 study in Norway. PLoS One 2012, 7(7):e42113.

23. Siezenga MA, Shaw PK, Daha MR, Rabelink TJ, Berger SP: Low MannoseBinding Lectin (MBL) genotype is associated with future cardiovascular events in type 2 diabetic South Asians. A prospective cohort study. Cardiovasc Diabetol 2011, 10:60.

24. Mellbin LG, Hamsten A, Malmberg K, Steffensen R, Ryden L, Ohrvik J, Hansen TK: Mannose-binding lectin genotype and phenotype in patients with type 2 diabetes and myocardial infarction: a report from the DIGAMI 2 trial. Diabetes Care 2010, 33(11):2451-6.

25. Satomura A, Endo M, Fujita T, Ohi H, Ohsawa I, Fuke Y, Matsumoto K, Sudo S, Matsushita M, Fujita T: Serum mannose-binding lectin levels in maintenance hemodialysis patients: impact on all-cause mortality. Nephron Clin Pract 2006, 102(3-4):C93-c99.

26. Bay JT, Sorensen SS, Hansen JM, Madsen HO, Garred P: Low mannosebinding lectin serum levels are associated with reduced kidney graft survival. Kidney Int 2013, 83(2):264-71.

27. Libetta C, Rampino T, Palumbo G, Esposito C, Dal CA: Circulating serum lectins of patients with IgA nephropathy stimulate IL-6 release from mesangial cells. J Am Soc Nephrol 1997, 8(2):208-13.

28. Henry RM, Ferreira I, Kostense PJ, Dekker JM, Nijpels G, Heine RJ, Kamp O, Bouter LM, Stehouwer CD: Type 2 diabetes is associated with impaired endothelium-dependent, flow-mediated dilation, but impaired glucose metabolism is not. The Hoorn Study. Atherosclerosis 2004, 174(1):49-56.

doi:10.1186/1471-2369-15-162

Cite this article as: Hornum et al:: High levels of mannose-binding lectin are associated with lower pulse wave velocity in uraemic patients. BMC Nephrology 2014 15:162.

\section{Submit your next manuscript to BioMed Central and take full advantage of:}

- Convenient online submission

- Thorough peer review

- No space constraints or color figure charges

- Immediate publication on acceptance

- Inclusion in PubMed, CAS, Scopus and Google Scholar

- Research which is freely available for redistribution 